\title{
Obtention de présure à partir de veaux fistulés
}

\author{
par \\ F. CISNEROS* \\ (avec la collaboration de Caridad SILVA) \\ E. VALLES et G. MOCQUOT** \\ (avec la collaboration de J.P. FURET) \\ et \\ R. TOMASSONE***
}

\section{INTRODUCTION}

Au cours des récentes années, la pénurie mondiale de caillettes de veaux de lait destinées à la fabrication de la présure a conduit les chercheurs à reprendre les premiers travaux de Fomin [1] en 1939 et ceux de Berridge [2] en 1943 concernant l'obtention de présure à partir de jus gastrique de veaux fistulés. Plusieurs publications ont fait état de recherches sur ce sujet [3-6].

Le présent travail rend compte des résultats obtenus à Cuba concernant l'obtention, à partir de veaux fistulés, de jus gastriques destinés à la préparation de la présure.

\section{MATERIEL ET METHODES}

Sur 10 veaux de race Holstein âgés de $12 \mathrm{j}$ environ, une canule a été placée dans la caillette selon la technique décrite par Nair, Mehar et Bhalerao [4]. Les veaux ainsi fistulés étaient logés pendant toute la durée de l'essai dans des cages spécialement aménagées pour réduire au minimum les risques d'arrachement de la canule et pour faciliter la récolte des jus gastriques.

* Centre National de la Recherche Scientifique (C.N.I.C.), La Havane (Cuba).

** Laboratoire de Recherches sur les Protéines (C.N.R.Z. - I.N.R.A.).

*** Laboratoire de Biométrie (C.N.R.Z. - I.N.R.A.), Jouy-en-Josas (France). 
TABLEAU 1. - Alimentation des veaux fistulés

\begin{tabular}{l|c|c}
\hline Age & $\begin{array}{c}\text { Quantité totale de lait } \\
\text { ingérée par jour } \\
\text { (en litres) }\end{array}$ & $\begin{array}{c}\text { Quantité } \\
\text { de lait ingérée par repas } \\
(\text { en litres })^{* *}\end{array}$ \\
\hline $10-15$ jours & 5 & 2,5 matin et soir \\
1 mois & 8 & 4,0 matin et soir \\
3 mois & 11 & 5,5 matin et soir \\
6 mois & 16 & 8,0 matin et soir \\
\hline * Ce régime est complété par l'ingestion des divers sels minéraux que les \\
veaux prennent à volonté en léchant des blocs constitués par ces sels. \\
* Le lait est ingéré aussitôt après le prélèvement de jus gastrique. \\
\hline
\end{tabular}
ration.

La récolte des jus gastriques commençait 8 à $10 \mathrm{j}$ après l'opé-

\section{Alimentation des animaux et récolte des jus gastriques}

La récolte des jus gastriques était pratiquée deux fois par jour : le premier prélèvement avait lieu à $8 \mathrm{~h}$ et le deuxième à $16 \mathrm{~h}$. Aussitôt après chaque prélèvement les veaux étaient nourris au lait entier selon le régime indiqué dans le tableau 1 . Le prélèvement de jus gastrique était pratiqué selon les deux méthodes mentionnées au tableau 2 qui donne également le plan de l'expérience.

\section{Détermination de l'activité coagúlante et du pH}

Le volume des différents jus gastriques prélevés à partir de chaque veau était d'abord mesuré. Les échantillons étaient ensuite placés dans un réfrigérateur entre $3^{\circ} \mathrm{C}$ et $5^{\circ} \mathrm{C}$ jusqu'au lendemain matin, puis transportés au laboratoire. Les jus 1, 2 et 3 (méthode a) du matin étaient mélangés avec les jus 1,2 et 3 de l'après-midi. La même opération était pratiquée pour les jus 1 et 3 dans la méthode $b$.

Les jus ainsi mélangés étaient ensuite filtrés sur gaze afin de les débarrasser des particules grossières`(résidus de lait caillé, poils, etc.). Ils étaient portés à la température de $20^{\circ} \mathrm{C}$ et le $\mathrm{pH}$ était déterminé à l'aide d'un pH-mètre LEI-TZU modèle 25 (précision 0,05 unité $\mathrm{pH})$.

L'activité coagulante était déterminée par la méthode de Berridge avec les modifications introduites par Valles et Mocquot [7]. 
TABLEAU 2. - Méthodes d'obtention de jus gastriques

\begin{tabular}{|c|c|c|c|c|}
\hline \multirow{2}{*}{$\begin{array}{c}\text { Méthodes } \\
\text { de } \\
\text { prélèvement }\end{array}$} & \multicolumn{2}{|c|}{ Heures (h) des } & \multirow{2}{*}{ Veaux } & \multirow{2}{*}{$\begin{array}{l}\text { Durée de } \\
\text { l'essai } \\
\text { (en mois) }\end{array}$} \\
\hline & Prélèvements & Repas & & \\
\hline $\begin{array}{l}\text { Méthode a } \\
\text { Temps "zéro " : } \\
\text { Récolte du jus } 1 \text { (à jeun) } \\
\text { Ingestion de } 250 \text { ml de lac- } \\
\text { tosérum* dilué et aussitôt } \\
\text { après : } \\
\text { Récolte du jus } 2 \\
20 \mathrm{mn} \text { après : } \\
\text { Récolte du jus } 3\end{array}$ & 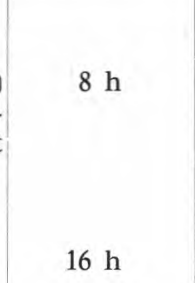 & $17 \mathrm{~h}$ & $\begin{array}{l}\mathrm{C} \\
\mathrm{H} \\
\mathrm{I} \\
\mathrm{J}\end{array}$ & $\begin{array}{l}6 \\
6 \\
4^{* *} \\
3^{* * *}\end{array}$ \\
\hline $\begin{array}{l}\text { Méthode b } \\
\text { Temps "zéro ": } \\
\text { Récolte du jus 1 (à jeun) } \\
\text { Ingestion de } 2,51 \text { de las- } \\
\text { tosérum dilué et } 40 \mathrm{mn} \\
\text { plus tard : } \\
\text { Récolte du jus } 3\end{array}$ & $16 \mathrm{~h}$ & $17 \mathrm{~h}$ & $\begin{array}{l}\text { C } \\
\text { D } \\
\text { E } \\
\text { F } \\
\text { K } \\
\mathrm{K}^{\prime}\end{array}$ & $\begin{array}{l}6 \\
4^{* \star *} \\
6 \\
6 \\
6 \\
3^{* \ldots *}\end{array}$ \\
\hline \multicolumn{5}{|c|}{$\begin{array}{l}\text { * Lactosérum obtenu par coagulation du lait entier par la présure à } 37^{\circ} \mathrm{C} \\
\text { en } 20 \mathrm{mn} \text { environ et dilué avec } 5 \text { parties d'eau. } \\
\text { ** Cause ayant entraîné le retrait de l'essai : asphyxie accidentelle. } \\
\text { ** Cause ayant entraîné le retrait de l'essai : hernie. }\end{array}$} \\
\hline
\end{tabular}

TABLEAU 3. - Influence de la méthode de prélèvement

\begin{tabular}{|c|c|c|c|}
\hline Méthode & Jus gastriques & $\begin{array}{l}\text { Volume* } \\
\text { (en ml) }\end{array}$ & $\begin{array}{c}\text { Quantité de présure* } \\
\text { (en UP) }\end{array}$ \\
\hline$a$ & $\begin{array}{cc} & \text { jus } 1 \\
& \text { jus } 2 \\
& \text { jus } 3 \\
& \text { jus } 2+\text { jus } 3 \\
\text { jus } 1+\text { jus } 2+\text { jus } 3\end{array}$ & $\begin{array}{r}808 \\
587 \\
362 \\
949 \\
1757\end{array}$ & $\begin{array}{r}6164 \\
3264 \\
3216 \\
6480 \\
12644\end{array}$ \\
\hline$b$ & $\begin{array}{c}\text { jus } 1 \\
\text { jus } 3 \\
\text { jus } 1+\text { jus } 3\end{array}$ & $\begin{array}{l}1219 \\
1134 \\
2353\end{array}$ & $\begin{array}{r}10851 \\
7273 \\
18123\end{array}$ \\
\hline $\begin{array}{l}\text { Valeurs } \\
\text { période }\end{array}$ & $\begin{array}{l}\text { yennes journalières } \\
\text { mois. }\end{array}$ & au ca & our l'ensemble de la \\
\hline
\end{tabular}




\section{RESULTATS ET DISCUSSION}

L'objectif essentiel de cette étude était de comparer les méthodes $a$ et $b$ et d'étudier la variabilité individuelle et l'influence de l'âge sur les volumes de jus gastriques récoltés et sur les quantités de présure obtenues.

Nous avons utilisé une méthode statistique classique, l'analyse de covariance [8] permettant de déterminer si les résultats obtenus avec chaque méthode étaient identiques ou non et, en outre, de chiffrer par le calcul d'un écart type résiduel la variabilité propre à tout phénomène biologique.

Le nombre de jours de prélèvement ayant été différent pour les deux méthodes étudiées, nous en avons tenu compte en introduisant une légère correction.

\section{Influence des méthodes de prélèvement}

Le tableau 3 montre les résultats obtenus avec les deux méthodes. Les valeurs moyennes journalières, calculées sur l'ensemble des 6 mois d'étude montrent des différences significatives $(P=0,05)$ aussi bien pour les volumes de jus gastriques récoltés que pour leurs activités coagulantes.

Ces résultats montrent que la méthode $b$ s'est avérée plus efficace que la méthode $a$. En effet, elle conduit à l'obtention d'une quantité de présure environ 1,4 fois plus élevée et cette différence est dûe à une concentration en présure plus grande des jus gastriques, notamment du jus 3 , comme le montre le tableau 4 .

Le $\mathrm{pH}$ du jus gastrique est indépendant des méthodes de prélèvement utilisées et il est suffisamment bas pour activer la proprésure des jus gastriques (tab. 4).

TABLEAU 4. - Influence de la méthode de prélèvement

\begin{tabular}{|c|c|c|c|}
\hline Méthodes & Jus gastriques & $\begin{array}{l}\text { Concentration en présure } \\
(\text { en UP } / \mathrm{ml})\end{array}$ & $\mathrm{pH}$ \\
\hline$a$ & $\begin{array}{l}\text { jus } 1 \\
\text { jus } 3\end{array}$ & $\begin{array}{l}8,2 \\
6,7\end{array}$ & $\begin{array}{l}2,3 \\
2,4\end{array}$ \\
\hline$b$ & $\begin{array}{l}\text { jus } 1 \\
\text { jus } 3\end{array}$ & $\begin{array}{l}7,9 \\
9,8\end{array}$ & $\begin{array}{l}2,5 \\
2,4\end{array}$ \\
\hline $\begin{array}{l}\text { Valeurs } \\
\text { période }\end{array}$ & $\begin{array}{l}\text { s journalières } \\
\text { s. }\end{array}$ & veau calculées pour l' & le de la \\
\hline
\end{tabular}


TABLEAU 5. - Influence de la variabilité individuelle

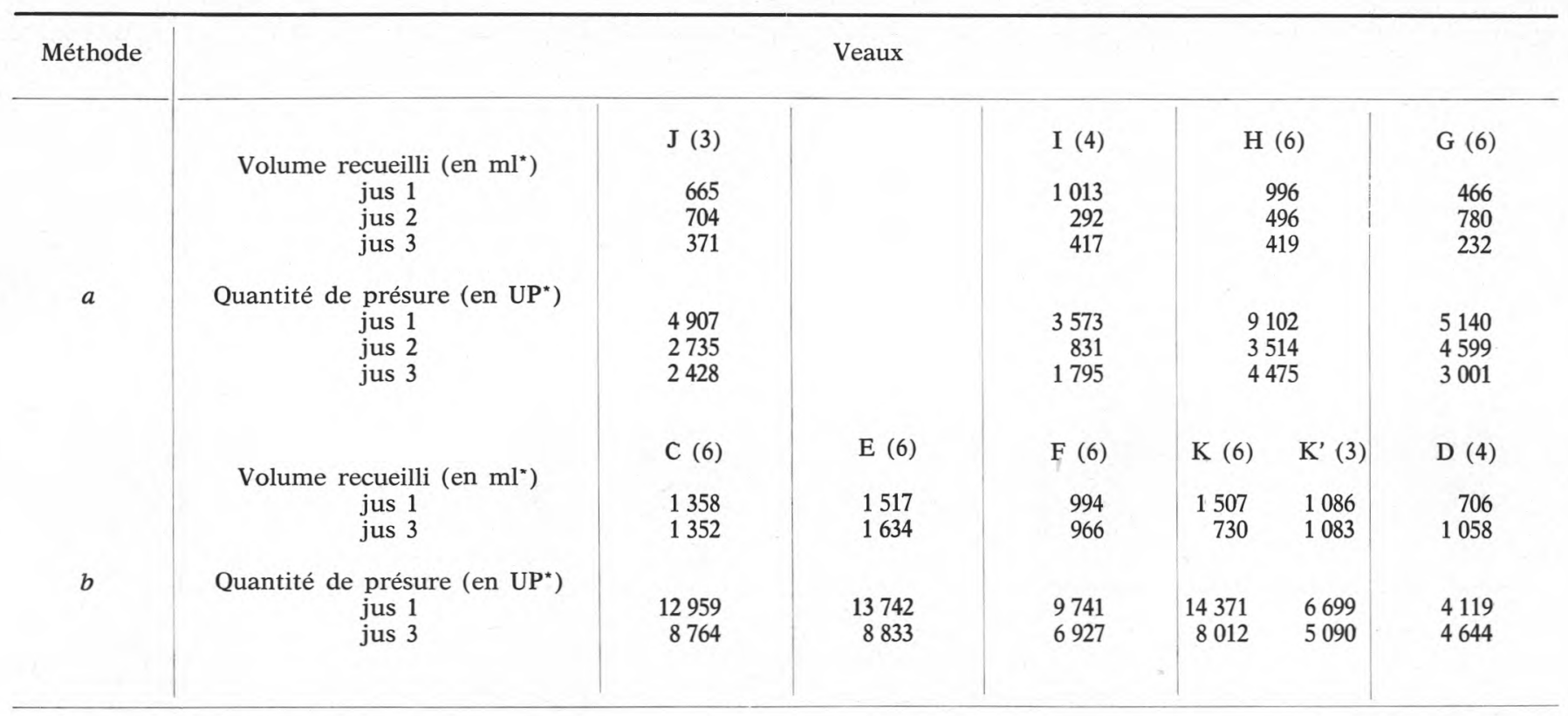

* Moyennes journalières.

Les chiffres entre parenthèses indiquent les nombres de mois sur lesquels les moyennes journalières ont été établies. 


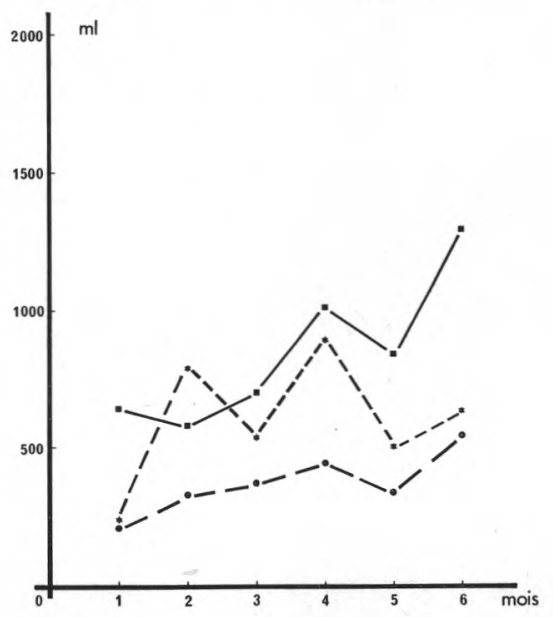

fig. 1

Votume de jus gastrique recueilli en fonction de l'âge* Méthode a

- - Jus 1

$\star \ldots \ldots$ Jus 2

Jus 3

Moyenne journalière d'après les résultats de tous les prélèvements d'un mois.

Valeurs établies : $1^{\text {er }}, 2^{\text {me }}$ et $3^{\text {me }}$ mois sur 4 veaux. $4^{\text {me }}$ mois sur 3 veaux.

$5^{\text {me }}$ et $6^{\text {me }}$ mois sur 2 veaux.

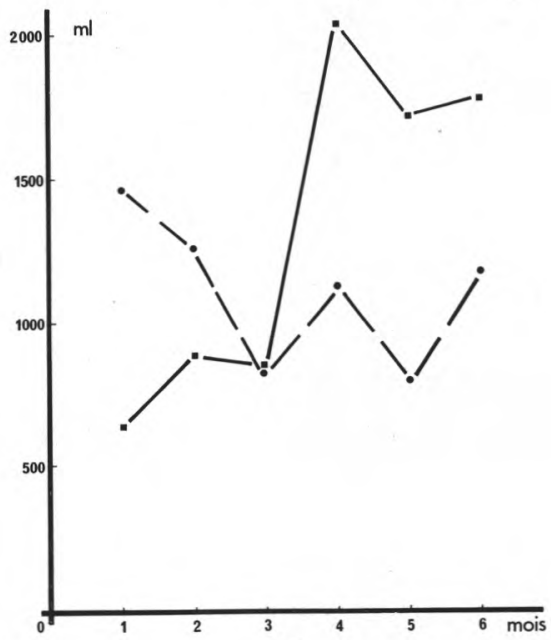

fig. 2

Volume de jus gastrique recueilli en fonction de l'âge*

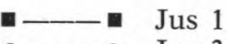
Méthode b

-.._- Jus 3

Moyenne journalière par veau d'après les résultats de tous les prélèvements d'un mois.

Valeurs établies : $1^{\text {er }}, 2^{\text {me }}$ et $3^{\text {tme }}$ mois sur 6 veaux. $4^{\text {me }}$ mois sur 5 veaux. $5^{\text {me }}$ et $6^{\text {me }}$ mois sur 4 veaux. 


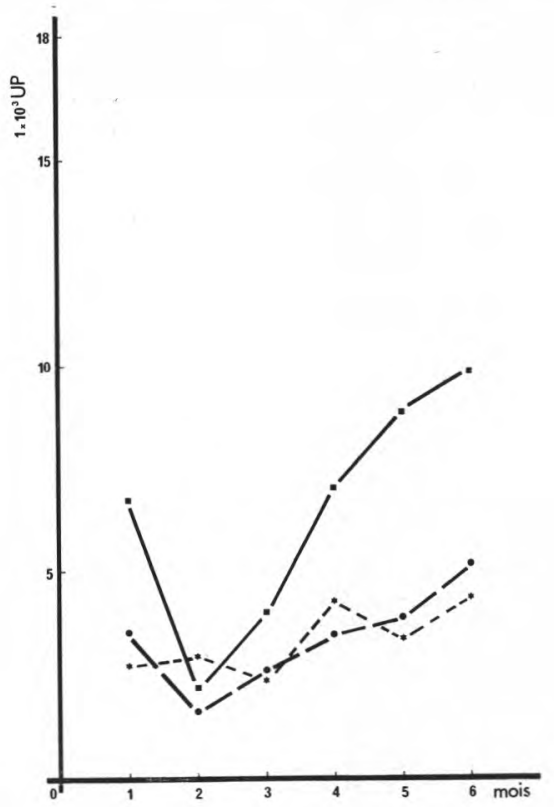

fig. 3

Quantité de présure obtenue en fonction de l'âge* Méthode a

Jus 1

$\star \ldots \ldots$ Jus 2

-_- Jus 3

* Moyenne journalière par veau d'après les résultats de tous les prélèvements d'un mois.

Valeurs établies : $1^{\text {er }}, 2^{\text {me }}$ et $3^{\text {me }}$ mois sur 4 veaux.

$4^{\text {me }}$ mois sur 3 veaux.

$5^{\text {me }}$ et $6^{\text {me }}$ mois sur 2 veaux.

\section{Influence de la variabilité individuelle}

Le tableau 5 montre les valeurs individuelles obtenues avec les méthodes $a$ et $b$. Ces résultats montrent une très grande variabilité des volumes récoltés. Par exemple, les valeurs extrêmes pour la méthode a vont de $232 \mathrm{ml}$ à $419 \mathrm{ml}$ de jus gastrique par jour (moyenne $=362 \mathrm{ml}$ ) et de 730 à $1634 \mathrm{ml}$ (moyenne $=1134 \mathrm{ml}$ ) pour la méthode $b$.

Ces variations sont d'une importance capitale et il conviendra d'en tenir compte pour la planification statistique des futures expériences. Ainsi pour le jus 1 de la méthode $a$, l'écart type résiduel des mesures de volume est de $249 \mathrm{ml}$ et, pour le jus 1 de la méthode $b$, cet écart type résiduel est de $357 \mathrm{ml}$. 


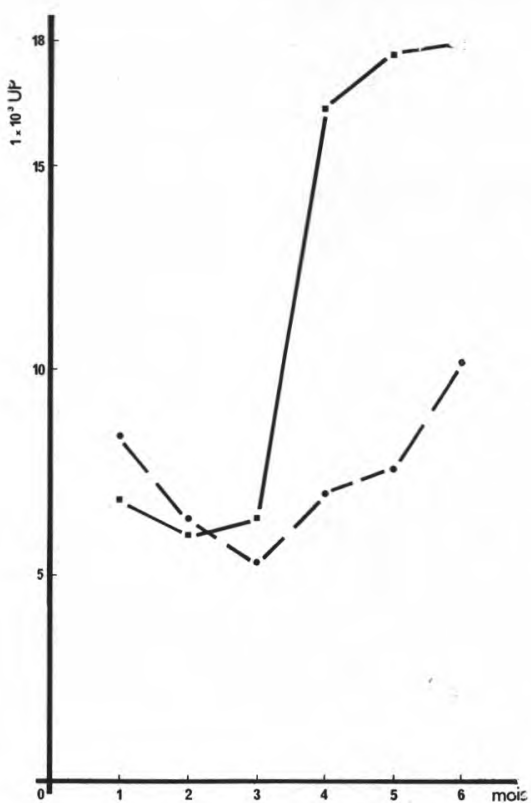

fig. 4

Quantité de présure obtenue en fonction de l'âge* Méthode b

- Jus 1

-——— Jus 3

* Moyenne journalière par veau d'après les résultats de tous les prélèvements d'un mois.

Valeurs établies : $1^{\text {er }}, 2^{\text {me }}$ et $3^{\text {me }}$ mois sur 6 veaux.

$4^{\text {me }}$ mois sur 5 veaux.

$5^{\mathrm{me}}$ et $6^{\mathrm{me}}$ mois sur 4 veaux.

\section{Influence de l'âge}

Les figures 1 et 2 montrent la variation du volume de jus gastrique récolté en fonction de l'âge et les figures 3 et 4 , celles correspondant aux quantités de présure qui ont été obtenues avec les deux méthodes de prélèvement.

L'analyse statistique a montré que les variations constatées avec la méthode a ne sont pas significatives (fig. 1 et 3 ). Par contre, les variations obtenues avec la méthode $b$, (fig. 2 et 4) peuvent être considérées comme significatives. Avec la méthode $b$ on constate pour le jus 1 une très nette augmentation du volume entre le $3^{\text {me }}$ et le $4^{\text {me }}$ mois, suivie d'un palier jusqu'au $6^{\text {me }}$ mois. Cette augmentation est d'ailleurs très voisine pour la quantité de présure. Pour le jus 3 le volume varie peu, mais la quantité de présure passe par un minimum au $3^{\text {me }}$ mois et augmente ensuite régulièrement. Il semblerait 


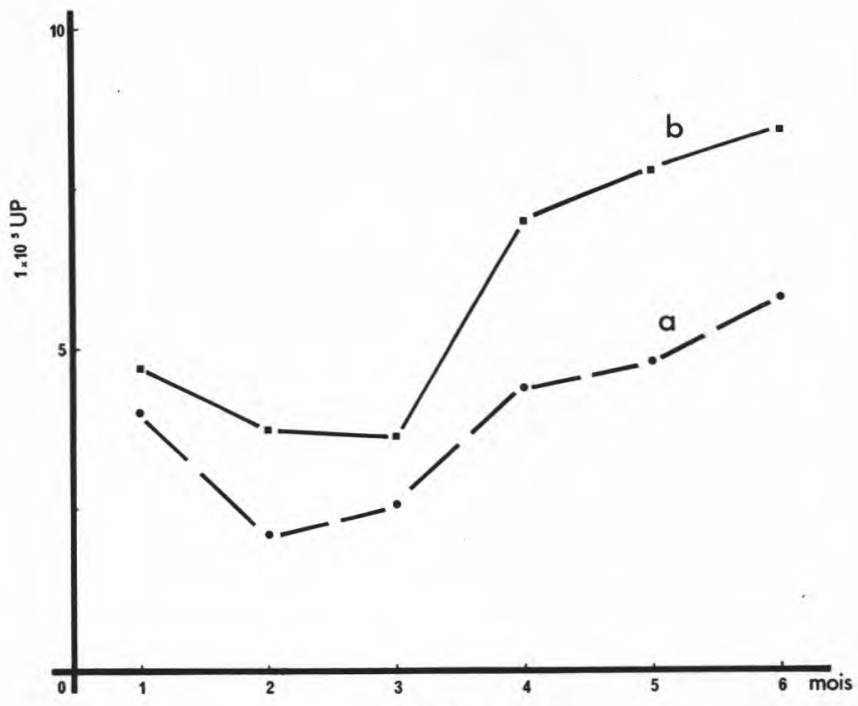

fig. 5

Quantité de présure obtenue en fonction de l'âge * -_- Méthode a

Moyenne des quantités mensuelles par veau.

Méthode $a$ :

Valeurs établies : $1^{\mathrm{er}}, 2^{\mathrm{me}}$ et $3^{\text {me }}$ mois sur 4 veaux.

$4^{\text {me }}$ mois sur 3 veaux.

Méthode $b$ :

$5^{\text {me }}$ et $6^{\text {me }}$ mois sur 2 veaux.

Valeurs établies : $1^{\mathrm{er}}, 2^{\mathrm{me}}$ et $3^{\text {me }}$ mois sur 6 veaux.

$4^{\text {me }}$ mois sur 5 veaux.

$5^{\text {me }}$ et $6^{\text {me }}$ mois sur 4 veaux.

donc que l'influence de l'âge soit différente selon la méthode de prélèvement de jus gastrique utilisée. Néanmoins il faut remarquer que les valeurs dans les figures 1 et 3 ont été établies à partir d'un lot de 4 veaux dont 2 seulement ont été en essai pendant 6 mois, tandis que pour la méthode $b$, les valeurs des figures 2 et 4 ont été obtenues à partir d'un lot de 6 veaux dont 4 ont été en essai pendant la même période (tab. 2). On peut se demander alors si le fait de ne pas avoir trouvé de différences significatives, dans le cas de la méthode $a$, entre les volumes des différents jus gastriques et les quantités de présure correspondantes en fonction de l'âge, n'est pas dû au faible nombre d'animaux en expérience (deux) jusqu'au $3^{\text {me }}$ et $6^{\text {me }}$ mois. Autrement dit, on ne peut préciser si le manque d'influence significative de la méthode a sur les variations des volumes et des quantités de présure en fonction de l'âge est dû à la méthode elle-même ou au nombre de veaux, très restreint, mis en essai. 
La figure 5 montre enfin les quantités moyennes de présure obtenues par veau et par mois au moyen des deux méthodes de prélèvement, pendant la durée de l'essai. Ces courbes indiquent encore la supériorité de la méthode $b$ sur la méthode $a$ et, en même temps, elles indiquent qu'au bout de 6 mois la production de présure par les veaux fistulés est encore en augmentation.

Dans le cas de la méthode $b$, la quantité totale de présure produite par un veau pendant 6 mois, est en moyenne de l'ordre de 3500000 UP. Or il est admis et nous l'avons nous-mêmes vérifié, que la caillette sèche d'un veau de lait abattu fournit en moyenne 50000 UP. On voit ainsi qu'un veau fistulé, maintenu en production de jus gastrique pendant 6 mois, produit en moyenne autant de présure que 70 caillettes de veaux de lait abattus.

\section{CONCLUSIONS}

Les résultats concernant l'obtention de jus gastrique à partir d'un lot de 10 veaux fistulés permettent, après une période de 6 mois d'essai de tirer les conclusions suivantes.

Il est possible de maintenir en production de jus gastrique des veaux fistulés alimentés exclusivement au lait entier pendant 6 mois. Sur un effectif de 10 veaux au départ, 6 ont été maintenus sans difficultés pendant tout l'essai. L'apparition de hernies chez 3 veaux sur 10 doit être considérée comme un problème lié aux suites de l'opération. Bien qu'une perte de l'ordre de 30 p. 100 puisse apparaître comme élevée, on peut estimer que la maîtrise de la technique opératoire pourrait devenir meilleure à la suite d'une application répétée sur un plus grand nombre d'animaux.

La méthode $b$ de prélèvement de jus gastrique est une méthode simple qui permet l'obtention de quantités importantes de jus gastriques assez riches en présure.

Dans les conditions décrites, la production de jus gastrique peut être conduite pendant une période d'au moins 6 mois sans diminution de la quantité de présure obtenue. Toutefois, il faudrait déterminer dans ces conditions quel est le rapport entre les teneurs en présure et pepsine des différents jus gastriques en fonction de l'âge des veaux. Il faudrait étudier également la qualité de différents types de fromages fabriqués à partir de la présure ainsi obtenue.

Il est encourageant de constater qu'un veau fistulé maintenu en production pendant 6 mois est capable de produire autant de présure que ce que l'on obtient à partir de 70 caillettes sèches. Ce résultat montre qu'il serait possible d'une part de multiplier par 70 la production de présure à partir d'un veau de lait au moyen de la tech- 
nique de la fistule abomasale et, d'autre part, que la présure obtenue peut rester compétitive avec la présure traditionnelle aussi longtemps que les frais d'exploitation par veau fistulé (pour une période de $180 \mathrm{j}$ de prélèvement à raison de deux récoltes de jus gastrique par jour) demeurent inférieurs à 70 fois le prix d'une caillette de bonne qualité. Bien entendu le gain résultant de la croissance du veau au cours de la même période doit être pris en ligne de compte pour ce calcul.

Néanmoins, ces premières conclusions doivent être étayées par de nouveaux essais, compte tenu de la variabilité individuelle et, d'autre part pour compléter l'étude d'autres facteurs (influence de la race, du nombre de récoltes journalières, etc.) susceptibles d'influencer la production de jus gastrique par le veau fistulé.

\section{R és u m é}

On a étudié la production de jus gastrique à partir d'un lot de 10 veaux fistulés de race Holstein nourris au lait entier. Deux méthodes de prélèvement du jus gastrique ont été expérimentées pendant 6 mois. On a étudié l'influence de l'âge, de la variabilité individuelle et de l'efficacité de chacune des méthodes utilisées sur la production de présure.

La méthode de prélèvement consistant à prélever le jus gastrique du veau à jeun et à faire ensuite ingérer par ce veau 2,5 1 de lactosérum dilué et à récolter le jus gastrique $40 \mathrm{mn}$ après l'ingestion a été retenue.

La production de présure augmente avec l'âge, notamment à partir du $3^{\text {me }}$ mois, elle est encore en augmentation au bout de 6 mois. La quantité totale de présure contenue dans les jus gastriques récoltés deux fois par jour pendant 6 mois à partir d'un veau fistulé, est équivalente à celle contenue dans 70 caillettes sèches de veaux de lait abattus.

Des variations individuelles assez importantes ont été remarquées parmi les 10 veaux étudiés.

\section{Remerciements}

Nous remercions vivement le $D r J$. Renaud de l'Institut National de Médecine Vétérinaire de Cuba qui a été chargé de l'opération des veaux. Nous remercions également $M$. Ribadeau-Dumas pour les conseils et suggestions qu'il nous a apportés au cours de ce travail, ainsi que pour la rédaction du manuscrit. 


\section{S u m m a r y}

The production of gastric juice from fistulated milk fed calves was studied during a 6 months period. The fistula operation was performed on the calves according to the technique of Nair et al.

The influence of age and individual variations on the production of rennet was studied using two procedures to collect the gastric juices. The procedure consisting in collection of gastric juice from the empty stomach, subsequent feeding of the calf with 2,5 liters of diluted whey and collection of the gastric juice $40 \mathrm{mn}$ later was the most efficient.

The production of gastric juice by fistulated calves increased with age as well as the production of rennet. The total number of rennin units obtained from one fistulated calf during a 6 months period, performing two extractions of gastric juice per day, was equivalent to 70 dried vells of slaughtered calves.

Important individual variations in the volumes obtained and in the rennin concentration of the gastric juices were observed among the 10 calves studied.

\section{Bibliographie}

[1] Fomin (D.) (1939). - The preparation of rennet from abomasum of living calves. Molochno-Maslodel'naya Prom. 6, (9), 18. Dans Dairy Sci. Abst., (1941-1942), 3, 16.

[2] Berridge (N. J.), Davis (J. G.), Kon (P. M.), Kon (S. K.) and Spratling (F. R.) (1943). - The production of rennet from living calves. J. Dairy Res., $13,143$.

[3] CRosskopf (J.F.W.) (1959). - Some factors affecting the secretion of abomasal juice in young dairy calves. Onderst. J. of Vet., 28, 133.

[4] NaIR (P. G.), Mehar Singh and Bhalerao (V.R.) (1963). - The operation for collecting rennet through an abomasal fistula. Indian Vet. J., 42, 250.

[5] Mathur (M. P.), Nair (C. K.), Walli (T. K.), Nath (I.) and Ganguli (N. C.) (1970). - Etude sur les possibilités de préparation de présure à partir d'animaux fistulés. XVIII $I^{\mathrm{e}}$ Congr. Int. Lait., $1 \mathrm{~F}, 298$.

[6] Mathur (M. P.), Prabhakaran (R. J. V.), Nair (P. G.) and Bhalerao (V. R.) (1970). - Rennet from buffalo and cow fistulated calves. Indian J. Dairy Sci., 23, 252.

[7] Valles (E.) et Mocouot (G.) (1972). - Etude sur la technique de préparation de la présure utilisée dans les fabrications traditionnelles des fromages de Gruyère de Comté et d'Emmental. Le Lait, Tome LII, n 515-516, 259.

[8] Pearce (S. G.) (1967). - Biological Statistics : An introduction. Mc Graw Hill, London. 